\title{
Observations on Time 2012
}

\author{
Sai Prahlad K
}

\begin{abstract}
The main purpose of this paper is to present the author's observations on time to you, the world. Time has been a major subject of religion, philosophy, and science but defining it in a non-controversial manner applicable to all fields of study has consistently eluded the greatest scholars. Therefore, the goal of this paper is to define time in a new way that has never been presented before. The potential bombshell in the paper is the unification of thermodynamics with general relativity.
\end{abstract}

\section{Introduction:}

Time is best observed by change, a change in physical or chemical process. What is change? And what causes change?

From a scientific perspective, Change is caused by a Force. Example: The Electromagnetic force, the gravitational force, the strong nuclear force and the weak nuclear force. A change in position of an object is Motion; it can also be described as velocity, acceleration, displacement and time. Motion is observed by attaching a frame of reference to a body and measuring its change in position relative to another reference. As there is no absolute frame of reference absolute motion cannot be determined. Everything in the universe can be considered to be moving (in a state of motion). An object's motion cannot change unless it is acted upon by a force.

Force Causes -> Motion -> motion causes -> Change

In reality, motion and change are like two sides in a coin; easily distinguishable but the same.

Observations from our everyday lives:

\section{Method:}

\section{Observation A}

1. Take a clock

2. Observe the changes happening in the clock, and note the passing of time.

3. Time period 15 seconds to 1 hour.

\section{Observation B}

1. Take an electronic gadget, preferably a laptop or a mobile phone

2. Keep the device on, till it runs out of battery

3. Note the changes and causes that lead the gadget to run out of electricity

Observation C

1. Take the sun

2. Observe its changes and note down the process

3. Time period for 2 days or a week.

\section{Observation D}

1. Take a living organism, preferably ourselves

2. Observe the changes that are happening in a day, and ask your-self the question why do we need food, water and oxygen for survival? What is the reason?

\section{Observation E}

1. Take the Human eyes (not literally) or any photoreceptors, including telescopes, Binoculars etc.

2. Observe and ask the question: why are we able to see? What is the process?

3. Note down your conclusions

Observation F (for physicists)

1. Take the Effect of Gravitational time dilation

2. Write down your observations on why and how gravity affects time

3. Note the changes that are accruing in the object you're observing in differing gravitational potentials. Example: An object inside a black hole or above earth's atmosphere etc.,

\section{Observation A}

\section{Author's results and Discussions:}

When time passes: energy is released from the clock, by the means of sound (tick, tick, tick) and as photons (thermal radiation). This release of energy is the change that was happening in the clock when time was flowing forward. 


\section{Observation B}

When time passes: Energy is being released into the universe (from the laptop). The energy that was released lradiated away from the laptop is thermal radiation, photons, and sound. Due to this constant release of energy the laptop ran out of battery. The same is true for any electronic gadget. And this is also why we needconstant supply of electricity for our cool gadgets and luxury items to perform work.

\section{Observation C}

When time passes/when time flows forward: energy is being released, in this case from the sun to the universe. Note: Energy is released whenever an object performs an action or work. Stars perform work 24/7 till the end of their lives. Therefore, it is no coincidence that stars too release energy constantlyin the universe; and it is only because of this constant release of energy we are able to receive the sunlight, continuously. If energy is released randomly then the sunlight we receive here on earth will also be random. Known Facts: A) The sun loses 1 billion kilograms (2.2 billion pounds) of weight each second;B)Enough energy reaches the earth from the sun every second to fulfill all our power needs for a year.

\section{Observation D}

A living organism is like a machine. It cannot stay idle, if it is alive. The Human body for example performs work continuously just like our sun. An adult human takes about 23,000 breaths per day. Our Heart beats about 35 million times a year. Our brain receives about 100 million pieces of information at any one moment from your eyes, nose, ears, skin and receptors inside our body. Theamount of electrical energy generated by our brain is enough to power a light bulb! Messages are sent to our brain at around 360 kilometers (224 miles) per hour. Our Stomach lining replaces itself every three days. Our body destroys or loses two and a half million red blood cells every second. Luckily, it creates more at the same rate! In one day, our blood travels 19,312 Kilometers (12,000 miles)! We release energy constantly. Therefore we need food, water and oxygen to survive (conservation Law).

\section{Observation E}

The Human eye is a complex optical system which collects light from the surrounding environment, regulates its intensity through a diaphragm, focuses it through an adjustable assembly of lenses to form an image, converts this image into a set of electrical signals, and transmits these signals to the brain through complex neural pathways that connect the eye via the optic nerve to the visual cortex and other areas of the brain.

This complex process so easily done by our body works because energy is released constantly from our surroundings. Example and fact: Think of a light beam. A light beam in a billionth of a second travels about a foot. Now, when you look at yourself in a mirror in the morning, you say to yourself, well that's me! Actually that is not you at all. You are looking at an image that was you a billionth of a second ago; because that is the time it takes for light to go from your face to the mirror and back to your eyes. Same thing for the moon; when you look at the moon you are not really looking at the moon as it is at all, you are looking at the moon as it was about a second ago.

If energy was not released from our surroundings: then the whole universe would become a perfect black body, more like a black hole. Fact with author's results: Information inside black holesis invisible to us because energy is not released from black-holes. Hence, time stops inside black holes.

Extra information: Scientists are working on a 'cloak of invisibility' that will hide objects by making light waves flow around them, like a water flowing around a rock in a river.

\section{Observation F}

Gravitational time dilation: It is the effect of time passing at different rates in regions of different gravitational potential; the lower the gravitational potential, the more slowly time passes. Albert Einstein originally predicted this effect in his theory of relativity and it has since been confirmed by test of general relativity. This has been demonstrated by noting that atomic clocks at differing altitudes (and thus different gravitational potential) will eventually show different times. The effects detected in such experiments are extremely small, with differences being measured in nanoseconds.

The Reason atomic clocks show different times in differing altitudes is because of gravity and the release of energy. This release of energy can also be called as thermal radiation. When the gravitational potential is low, energy is released at a slower rate, slowing down time. Example: Living in a planet. When the gravitational potential is high, energy is released at a faster rate, increasing time. Example: Living in a space station or zero $\mathrm{G}$ environments. 
This Release of energy from everything, does it exist? And is there proof?

It does exist and it is already a known scientific fact, but the information was perceived differently. Two main facts:

1. Thermal Radiation

2. Black body Radiation

\section{Thermal Radiation:}

All matter above absolute zero degree temperature releases thermal radiation. According to the third law of thermodynamics, it is impossible to reach absolute zero degree temperature, because a minimum amount of entropy (heat) always exists. Thereforeall matter and antimatter present in the universe release energy (thermal radiation).

\section{Black body Radiation:}

A perfect black body is a body that absorbs and stores information, indefinitely. As there is no perfect black bodies present in the universe: everything matter and antimatter release energy. This release of energy is commonly called as black body radiation. These two known facts or Laws of physics are the proofs to author's claim.

\section{Observation X}

So Far we know:

1. Energy is released constantly in the universe.

2. Release of energy causes change to a physical or to a chemical system; Change results to time. Release of energy causes "Change", which causes "Time".

3. Release of energy can also be described as Force

4. If energy is not released into the universe: the object becomes a perfect black body (more like a black hole). Ceasing all physical and chemical process to take place.

In this observation $\mathrm{X}$, we are going to look at the possibility if therelease of energy can be reversed in a given system. Let us take the observations ABC and E. Before we begin, you must know that whenever energy is released into the cosmos the process makes an impact. The impact is usually information stored by an observer. So trying to reverse an impact event could destroy the information stored by the observer.

A. If we reverse the energy releases that accrued in a clock. Then the clock would gain its energy back.It is like time travellthe decrease of entropy in a system.

B. Likewise, the laptop too will gain its energy back

C. The sun would become young and heavy, the fusion processes will be reversed,

D. (From Observation E) We would forget events because the photons are never absorbed in the first place.

Observation $\mathrm{X}$ is only hypothetical because it is impossible for us to decrease entropy in a system. Even if we did decrease entropy in one particular area of space, the entropy outside that space will compensate for it,literally making us impossible to time travel or decrease entropy.

\section{Conclusion:}

Based on all the observations and facts, the author deduces that Release of energy is time, more accurately "rate at which energy is released defines time", because it creates the flowlarrow of time. But this definition leads us to a new question: 'what causes energy to be released?' For this the author replies:Big bang theory states that everything (all matter and antimatter; which is pure energy) was contained in volume less than one trillionth the size of a point of a pin, meaning the size of an atom. From that point, the universe expanded to its present state. There are many theories to explain thisexpansion of the universe; personally author prefersdark energy as the culprit, and furthermore, he proposes dark energy as an opposite form of purelordinary energy. He arrives at this conclusion based on two facts: 1. Third law of thermodynamics which state that all matter and antimatter have heat, and 2. Universe is not in thermodynamicequilibrium i.e. temperature of the universe defers from place to place. If the universe had only matter and no space then such a universe would be unchangeable, in a state of complete equilibrium. But if the universe had space and matter (energy) then such a universe would be in constantchange, in a state of chaos and difference. Our universe has energy (matter) and space. Therefore, it is constantly changing. Space is like dark energy without it the universe would not have expanded, but because of it, the universe is still expanding. Since, everything we see and everything we are, is made up of matter $(E=\mathrm{MC} 2)$, energy is constantly being radiated away and this constant release of energy gives rise to Force, motion and time. 


\section{Journal Paper:}

\section{References:}

[1] A. Einstein, "Über das Relativitätsprinzip und die ausdemselbengezogenenFolgerungen", Jahrbuch der Radioaktivität und Elektronik 4, 411-462 (1907); English translation, in "On the relativity principle and the conclusions drawn from it", in "The Collected Papers", v.2, 433-484 (1989); also in H M Schwartz, "Einstein's comprehensive 1907 essay on relativity, part I", American Journal of Physics vol.45,no.6 (1977) pp.512-517; Part II in American Journal of Physics vol.45 no.9 (1977), pp.811-

Books: 817; Part III in American Journal of Physics vol.45 no.10 (1977), pp.899-902, see parts I, II and III.

[2] Planck, M. (1914). the theory of heat radiation. Masius, M. (transl.) (2nd ed.). P. Blakiston's Son \& Co. OL7154661M.

[3] Anne Rooney, 1001 Shocking Science Facts (Published 2008) ISBN: 978-1-84858-008-4

[4] Stephen Hawking, a brief history of time: from the big bang to black holes(Published 1988) ISBN: 978-0-553-10953-5

[5] "Eye, human"Encyclopædia Britannica. Encyclopedia Britannica Ultimate Reference Suite. Chicago: Encyclopædia Britannica, 2010.

[6] Carnot, Sadi,reflections on the motive power of fire (New York: Dover, 1960)

[7] Gardner, Martin, time travel and other mathematical bewilderments (New York: W. H. Freeman, 1988)

[8] Gott, J. Richard,time travel in Einstein's universe: the physical possibilities of travel through Time (New York: Houghton Mifflin, 2001)

[9] Kaku, Michio,physics of the Impossible: a scientific exploration into the world of phases, force fields, teleportation, and time travel (New York: Doubleday, 2008)

[10] Leff, Harvey S. and Andrew F. Rex, eds. Maxwell's demon 2: entropy, classical and quantum Information, computing (Bristol, UK: Institute of Physics, 2003)

[11] Polkinghorne, J. the quantum world (New York: Longman, 1984)

[12] Taylor, Edwin F. and John A. Wheeler.spacetime physics (New York: W. H. Freeman, 1992)

[13] Thorne, Kip S. black holes and time warps: Einstein's Outrageous Legacy (New York: W. W. Norton, 1994)

[14] Van Ness, H. C. understanding thermodynamics (New York: Dover,1969)

[15] Wheeler, John A. a journey into gravity and spacetime (New York: Scientific American Library, 1990)

[16] Carroll, Sean,from eternity to here (Published 2010) ISBN: 0-525-95133-4

[17] Feynman, Richard Phillips, the character of physical law (Cambridge: MIT Press, 1965)

\section{Science Magazine:}

[18] Catchpole, Heather (200-09-04). Cosmos Online - Verging on absolute zero

Referral persons: 1. Benjamin Schumacher, 2.Lorenzo Maccone, 3. Neil deGrasse Tyson, 4. Sean Carroll, 5.Michio Kaku. 